\title{
The Korean Society for Neuro-Oncology (KSNO) Guideline for WHO Grade II Cerebral Gliomas in Adults: Version 2019.01
}

\author{
Young Zoon Kim¹, Chae-Yong Kim², Chan Woo Wee ${ }^{3}$, Tae Hoon Roh ${ }^{4}$, Je Beom Hong ${ }^{5}$, Hyuk-Jin Oh${ }^{6}$, Seok-Gu Kang ${ }^{7}$, Shin-Hyuk Kang ${ }^{8}$,

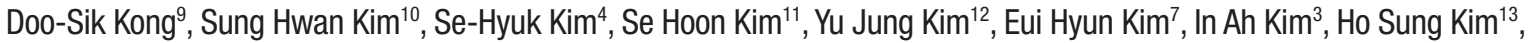 \\ Jae-Sung Park ${ }^{14}$, Hyun Jin Park ${ }^{15}$, Sang Woo Song ${ }^{16}$, Kyoung Su Sung ${ }^{17}$, Seung Ho Yang ${ }^{18}$, Wan-Soo Yoon ${ }^{19}$, Hong In Yoon ${ }^{20}$, \\ Jihae Lee ${ }^{21}$, Soon-Tae Lee ${ }^{22}$, Sea-Won Lee ${ }^{23}$, Youn Soo Lee ${ }^{24}$, Jaejoon Lim ${ }^{25}$, Jong Hee Chang ${ }^{7}$, Tae-Young Jung ${ }^{26}$, \\ Hye Lim Jung ${ }^{27}$, Jae Ho Cho ${ }^{20}$, Seung Hong Choi ${ }^{28}$, Hyoung Soo Choi ${ }^{29}$, Do Hoon Lim ${ }^{30 *}$, Dong-Sup Chung ${ }^{19 *}$; \\ KSNO Guideline Working Group
}

Received August 29, 2019

Revised September 2, 2019

Accepted September 30, 2019

\section{Correspondence}

Do Hoon Lim

Department of Radiation Oncology,

Samsung Medical Center,

Sungkyunkwan University School of

Medicine, 81 Irwon-ro, Gangnam-gu,

Seoul 06351, Korea

Tel: $+82-2-3410-2600$

Fax: +82-2-3410-2609

E-mail: dh8lim@skku.edu

Dong-Sup Chung

Department of Neurosurgery,

Incheon St. Mary's Hospital,

College of Medicine,

The Catholic University of Korea,

56 Dongsu-ro, Bupyeong-gu,

Incheon 21431, Korea

Tel: $+82-32-280-5871$

Fax: +82-32-280-5991

E-mail: dschung@catholic.ac.kr

*These authors contributed equally to this work as a corresponding author.
Background There was no practical guideline for the management of patients with central nervous system tumor in Korea for many years. Thus, the Korean Society for Neuro-Oncology (KSNO), a multidisciplinary academic society, has developed the guideline for glioblastoma. Subsequently, the KSNO guideline for World Health Organization (WHO) grade II cerebral glioma in adults is established.

Methods The Working Group was composed of 35 multidisciplinary medical experts in Korea. References were identified by searching PubMed, MEDLINE, EMBASE, and Cochrane CENTRAL databases using specific and sensitive keywords as well as combinations of keywords regarding diffuse astrocytoma and oligodendroglioma of brain in adults.

Results Whenever radiological feature suggests lower grade glioma, the maximal safe resection if feasible is recommended globally. After molecular and histological examinations, patients with diffuse astrocytoma, isocitrate dehydrogenase $(I D H)$-wildtype without molecular feature of glioblastoma should be primarily treated by standard brain radiotherapy and adjuvant temozolomide chemotherapy (Level III) while those with molecular feature of glioblastoma should be treated following the protocol for glioblastomas. In terms of patients with diffuse astrocytoma, IDH-mutant and oligodendroglioma (IDH-mutant and 1p19q codeletion), standard brain radiotherapy and adjuvant PCV (procarbazine+lomustine+vincristine) combination chemotherapy should be considered primarily for the high-risk group while observation with regular follow up should be considered for the low-risk group.

Conclusion The KSNO's guideline recommends that WHO grade II gliomas should be treated by maximal safe resection, if feasible, followed by radiotherapy and/or chemotherapy according to molecular and histological features of tumors and clinical characteristics of patients.

Key Words Korean Society for Neuro-Oncology; Guideline; Grade II Gliomas; Practice.

\section{INTRODUCTION}

Gliomas are the most common primary parenchymal brain tumors in the United States [1]. They are histologically diagnosed as the third most common primary tumor of the

This is an Open Access article distributed under the terms of the Creative Commons Attribution Non-Commercial License (https://creativecommons.org/licenses/by-nc/4.0) which permits unrestricted non-commercial use, distribution, and reproduction in any medium, provided the original work is properly cited.

Copyright $\odot 2019$ The Korean Brain Tumor Society, The Korean Society for NeuroOncology, and The Korean Society for Pediatric Neuro-Oncology central nervous system (CNS) in a relatively small portion in Korea [2]. According to the World Health Organization (WHO) classification of CNS tumors, the following tumors are classified as WHO grade II gliomas: diffuse astrocytoma, oligodendroglioma, pilomyxoid astrocytoma, pleomorphic xanthoastrocytoma, and ependymoma [3]. In fact, WHO grade II and III gliomas are not as common as glioblastomas. Other malignant gliomas including WHO grade II and III gliomas account for $10.2 \%$ and $5.3 \%$ of all primary CNS tumors in the United State [1] and Korea [2], respectively. 
The updated 4th edition of the WHO classification of CNS tumors was published in 2016. It had substantial changes compared to the previous edition of classification published in 2007. One of the most significant changes was the inclusion of both morphology and molecular features to have an integrated diagnosis for specific tumor entities [3,4]. In terms of diagnosis of glioma, there are several discrepancies in histological classification of gliomas due to considerable interobserver variability [5]. Recently, many studies using next-generation sequencing and microarray-based analyses have revealed characteristic genetic and epigenetic profiles of various types of gliomas [6]. Molecular biomarkers that may refine tumor diagnostics and improve prediction of treatment response and outcome have been identified [7]. These advancements in precise diagnoses are driven by widely available techniques such as immunohistochemistry, fluorescence in-situ hybridization (FISH), and DNA sequencing, and they make it possible to examine important molecular biomarkers for use with routinely formalin-fixed and paraffinembedded (FFPE) materials [6].

This paradigm shift was foreshadowed by the Haarlem recommendation that endorsed the incorporation of molecular biomarkers into pathologic diagnoses [4,8]. Major shifts in pathologic diagnosis of WHO grade II glioma included the use of molecular definitions for isocitrate dehydrogenase (IDH)-mutant astrocytomas, oligodendroglioma (IDH-mutant, $1 \mathrm{p} 19 \mathrm{q}$ codeleted) and $I D H$-wildtype astrocytomas, resulting in the elimination of oligoastrocytoma as an integrated diagnosis [3]. Additionally, the Consortium to Inform Molecular and Practical Approaches to CNS Tumor Taxonomy (cIMPACT-NOW) was established to enhance understanding the molecular pathogenesis of brain tumor warranting more rapid integration of this information into clinical practice between WHO updates. The cIMPACT-NOW recommends that the following genetic studies are the minimal molecular criteria for identifying an $I D H$-wildtype diffuse astrocytic glioma, although such $I D H$-wildtype diffuse astrocytic glioma appears histologically as a WHO grade II glioma known to have an aggressive clinical course and resemble $I D H$-wildtype glioblastoma: 1) epidermal growth factor receptor (EGFR) amplification, 2) combined whole chromosome 7 gain and whole chromosome 10 loss, and 3) telomerase reverse transcriptase (TERT) promoter mutation [9].

In terms of clinical course and outcome, compared to WHO grade III or IV gliomas, WHO grade II glioma has relatively favorable clinical outcomes. Its 5-year progressionfree survival (PFS) and overall survival (OS) rates have been reported to be $52.2 \%$ and $83.0 \%$, respectively, in Korea [10]. Its optimal primary treatment is surgical resection. Improved survival is expected after gross total resection [8]. Several clinical trials have examined effects of adjuvant radiotherapy including various dose escalation [11], optimal timing of radiotherapy [12], and chemotherapy-combined radiotherapy [13] on WHO grade II glioma. Adjuvant chemotherapy has also been explored as a potential replacement of radiotherapy for WHO grade II glioma [14]. Although maximal surgical resection as the primary therapeutic modality for WHO grade II glioma is the general consensus, adequate adjuvant treatment strategies have not been established yet.

Although the clinical practical guideline for grade IV gliomas has been developed and published recently by the Korean Society for Neuro-Oncology (KSNO), a multidisciplinary academic society for CNS tumors [15], an actual guideline for the management of patients with other CNS tumors in Korea is not available yet. The KSNO established a Working Group for developing practice guideline for CNS tumor in February 2018. The working group is recently trying to develop guidelines for CNS tumor based on updated information. The objective of KSNO guideline for WHO grade II cerebral gliomas is to provide physicians with evidence-based recommendations and consensus expert opinion for the management of patients with gliomas in daily clinical practice. It will also serve as a source of knowledge for institutions and insurance companies involved in cancer care in Korea.

\section{KSNO GUIDELINE WORKING GROUP}

A Working Group was appointed by the KSNO in February 2018 to establish guidelines for the management of glioblastoma patients. These guidelines should consider unique medical circumstance in Korea. The Working Group was composed of 35 medical experts in Korea, including 18 neurosurgeons, 8 radiation oncologists, 1 medical oncologist, 2 neuroradiologists, 3 pediatric oncologists, 2 pathologists, and 1 neurologist. As there is no medical specialty for neuro-oncology in Korea, neurosurgeon (especially brain tumor surgeon) usually plays a role of neuro-oncologist in clinical practice.

References were identified by searching PubMed, MEDLINE, EMBASE, and Cochrane CENTRAL databases using specific and sensitive keywords as well as combinations of these keywords. Scope of the disease was confined to cerebral diffuse astrocytoma and oligodendroglioma in adults aged $\geq 18$ years because other grade II gliomas are relatively uncommon in Korea. Therefore, grade II glioma in the spinal cord, pilomyxoid astrocytoma, ependymoma, and pleomorphic xanthoastrocytoma in the brain were excluded. Abstracts presented at official year-end conference of KSNO in December 2018 were considered relevant. If available, existing guidelines from national multidisciplinary neuro-oncological societies such as the National Comprehensive Cancer Network (NCCN) and European Association of Neuro-Oncology 


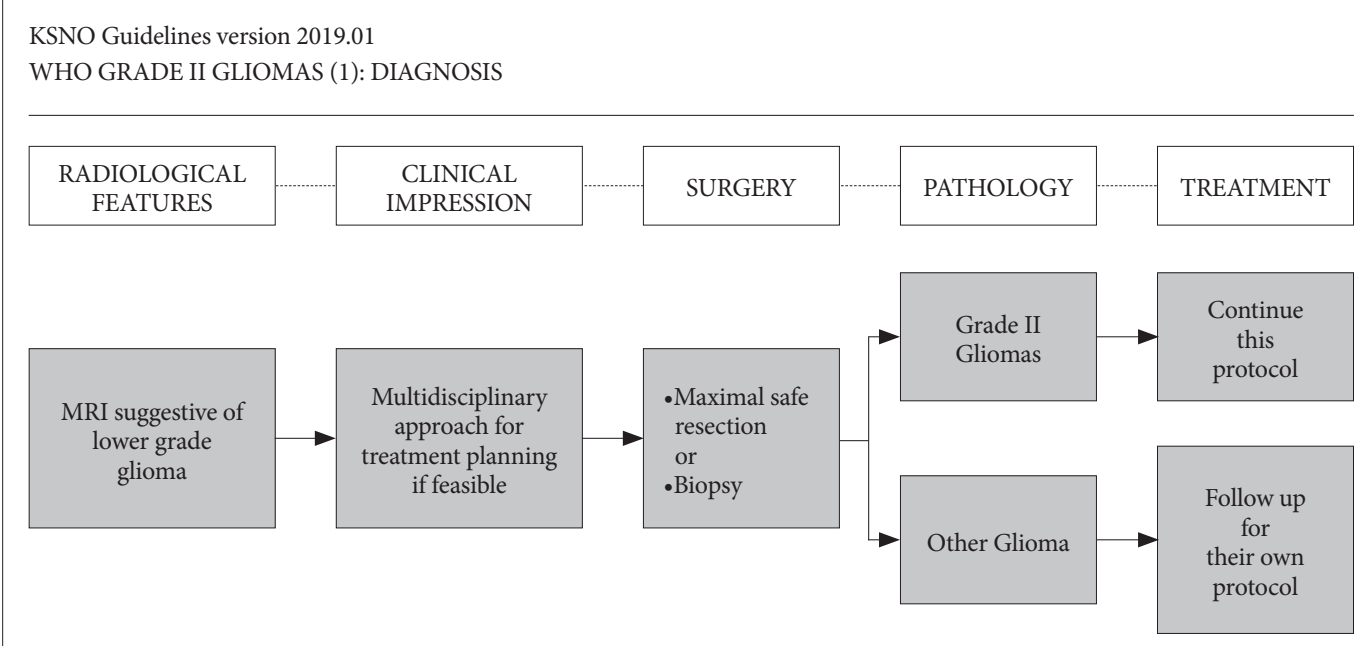

Fig. 1. Guideline for diagnosis of WHO grade II glioma. To diagnose lower grade gliomas, MRI with gadolinium enhancement is essential even if the lesions do not have enhancement. A multidisciplinary approach for treatment planning is recommended if feasible. Primarily, WHO grade II gliomas should be diagnosed by based on both histopathological and molecular genetic features of the tissues obtained by neurosurgical intervention. KSNO, Korean Society for Neuro-Oncology; MRI, magnetic resonance image.

(EANO) were also collected. The final reference list was generated based on originality and relevance to the scope of this guideline. The strategy of establishing this guideline was mainly based on NCCN and EANO guidelines with modifications and changes according to the unique background of Korea.

Scientific evidence was assessed and graded according to the following categories: high level of evidence (evaluated from multiple populations and derived from randomized clinical trials or meta-analysis or systemic review) and low level of evidence (evaluated from limited population and derived from non-randomized studies, including observational studies, cohort studies, and case-control studies).

To establish recommendation levels, the following criteria were used. Level I (strong recommendation) required a high level of evidence and uniform consensus among panels. Level II (weak recommendation) required a high level of evidence without uniform consensus among panels or low level of evidence but uniform consensus among panels. Level III (individual decision) required a low level of evidence without uniform consensus among panels. Level IV (not recommendable) required contents being not beneficial or harmful. Recommendations with level I and level II evidence were not marked. However, those with level III and level IV evidence were marked at the end of each recommendation in this guideline.

\section{DIAGNOSIS OF WHO GRADE II GLIOMAS}

When the radiological feature suggests a lower-grade glioma, multidisciplinary approach for treatment planning should be considered if feasible (Fig. 1). Computed tomography (CT) is insufficient to suggest a lower-grade glioma even with contrast enhancement. Therefore, magnetic resonance image (MRI) with contrast enhancement is essential to diagnose a lowergrade glioma. To obtain sufficient tissue for histopathological diagnosis, neurosurgical intervention is mandatory even if it is for stereotactic biopsy. To achieve maximal safe resection, neuronavigation systems, intraoperative $\mathrm{CT}$, MRI, intraoperative ultrasonography, intraoperative mapping techniques, and fluorescence-guidance with 5-aminolevuliniv acid are recommended. Histopathological diagnosis should be officially based on the 2016 WHO Classification of Tumors of the CNS [4].

Besides the importance of histopathological diagnosis of grade II glioma based on morphological features, codeletion of $1 \mathrm{p} 19 \mathrm{q}$ testing and IDH1/2 mutation testing are also essential parts in the molecular diagnosis for grade II glioma. ATPdependent helicase (ATRX) mutation test is also required for workup of grade II gliomas. If the tumor has IDH-wildtype, the following molecular tests are strongly recommended: 1) EGFR amplification, 2) combined whole chromosome 7 gain and whole chromosome 10 loss, and 3) TERT promoter mutation. MGMT promoter methylation test is also required for workup of all grade glioma.

\section{ADJUVANT TREATMENT OF WHO GRADE II GLIOMAS}

\section{Diffuse astrocytoma, $I D H$-wildtype}

In terms of histopathological and molecular features, diffuse astrocytoma, $I D H$-wildtype should be additionally analyzed with molecular test to determine glioblastoma features. The tumor is considered to have molecular features of glioblastoma if it has one or more of the following findings: 1) 


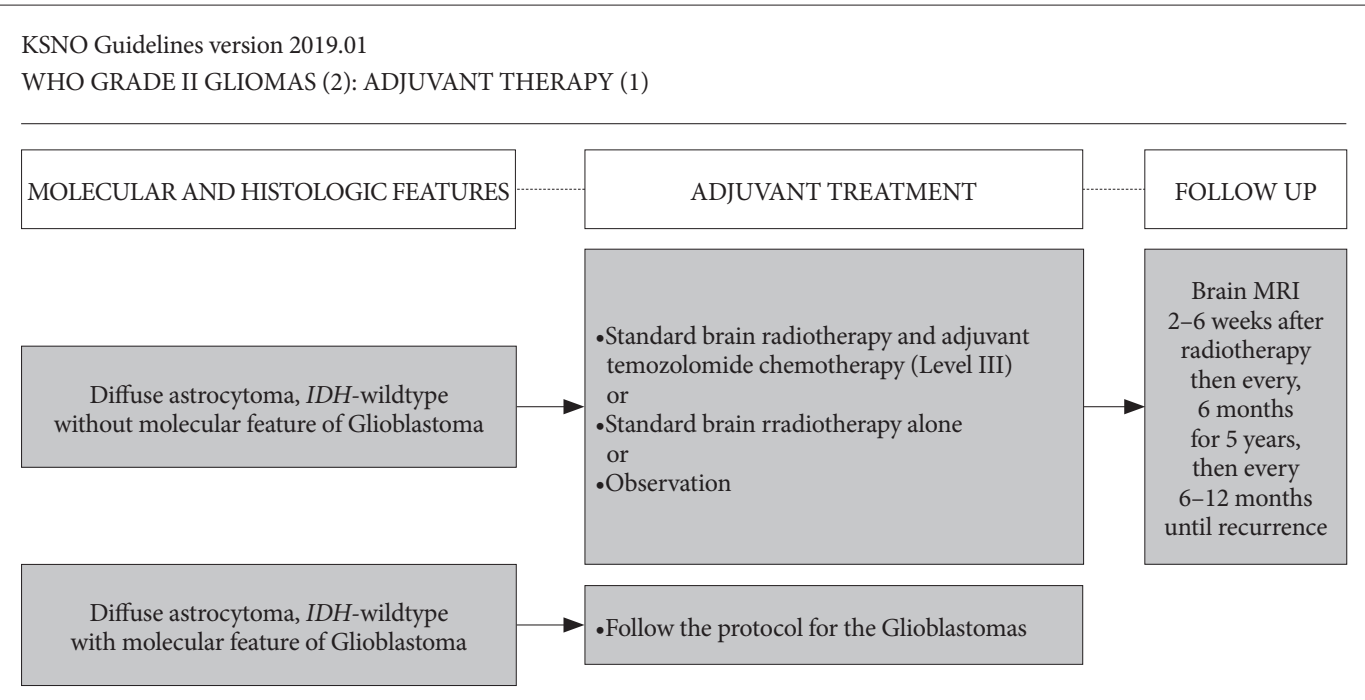

Fig. 2. Guideline for adjuvant therapy of patients with diffuse astrocytoma, $I D H$-wildtype. 1) Patients with diffuse astrocytoma, $I D H$-wildtype without molecular feature of glioblastoma should be primarily treated by standard brain radiotherapy and adjuvant temozolomide chemotherapy (Level III), 2) while those with molecular feature of glioblastoma should be treated following the protocol for glioblastomas. IDH, isocitrate dehydrogenase; KSNO, Korean Society for Neuro-Oncology; MRI, magnetic resonance image.

EGFR amplification, 2) combined whole chromosome 7 gain and whole chromosome 10 loss, and 3) TERT promoter mutation. If patients with diffuse astrocytoma, $I D H$-wildtype do not have molecular features of glioblastomas, they should be considered for the following modalities (Fig. 2): standard brain radiotherapy and adjuvant temozolomide chemotherapy (Level III), standard brain radiotherapy alone, or observation. However, if patients with diffuse astrocytoma, $I D H-$ wildtype have molecular feature of glioblastomas, they should be treated following the protocol for glioblastomas (Fig. 2). The dose of temozolomide is recommended to be 150-200 $\mathrm{mg} / \mathrm{m}^{2}$ for 5 sequential days every 4 weeks (Level III).

Radiological follow-up using MRI with gadolinium enhancement is recommended for regular check-up at 2-6 weeks after radiotherapy, then every 6 months for 5 years, and then every 6-12 months until recurrence of the disease (Fig. 2). It is necessary to follow up regularly with shorter interval for diffuse astrocytoma with $I D H$-wildtype than those with $I D H$ mutant because $I D H$-wildtype has worse prognostic feature.

\section{Diffuse astrocytoma, $I D H$-mutant and oligodendro- glioma (IDH-mutant, 1p19q codeletion)}

Patients who are older than 40 years and those who have not undergone gross total resection of the tumor are classified as the high-risk group. However, those who are younger than 40 years and those who have undergone gross total resection of the tumor are classified as the low-risk group. Although other risk factors such as tumor size and neurological deficit are considered, this guideline does not consider these factors for deciding the therapeutic modality.

For patients in the high-risk group, the following adjuvant treatment should be considered primarily: standard brain radiotherapy and adjuvant PCV (procarbazine, lomustine, and vincristine) combination chemotherapy, or concurrent chemoradiotherapy with temozolomide and adjuvant temozolomide chemotherapy, or standard brain radiotherapy with adjuvant temozolomide chemotherapy (Fig. 3). For patients in the low-risk group, the following adjuvant treatment should be considered: observation and regular follow-up, or standard brain radiotherapy alone, or adjuvant PCV (procarbazine, lomustine, and vincristine) combination chemotherapy alone (Fig. 3). The maximum number of cycles of adjuvant chemotherapy using PCV regimen (procarbazine, lomustine, and vincristine) is 6 . The recommended dose of temozolomide is $150-200 \mathrm{mg} / \mathrm{m}^{2}$ for 5 consequential days every 4 weeks for adjuvant purpose (Level III).

In terms of radiological follow-up, there is a uniform consensus among the panel for routine check of MRI at 2-6 weeks after radiotherapy with regular follow-up at 6-12 months interval consequentially until recurrence including progression. It is necessary to follow up regularly at longer interval for diffuse astrocytoma with $I D H$-mutant than for diffuse astrocytoma with $I D H$-wildtype because $I D H$-mutant has better prognostic feature.

\section{TREATMENT OF RECURRENT WHO GRDE II GLIOMAS}

It is more difficult to diagnose recurrences of WHO grade 


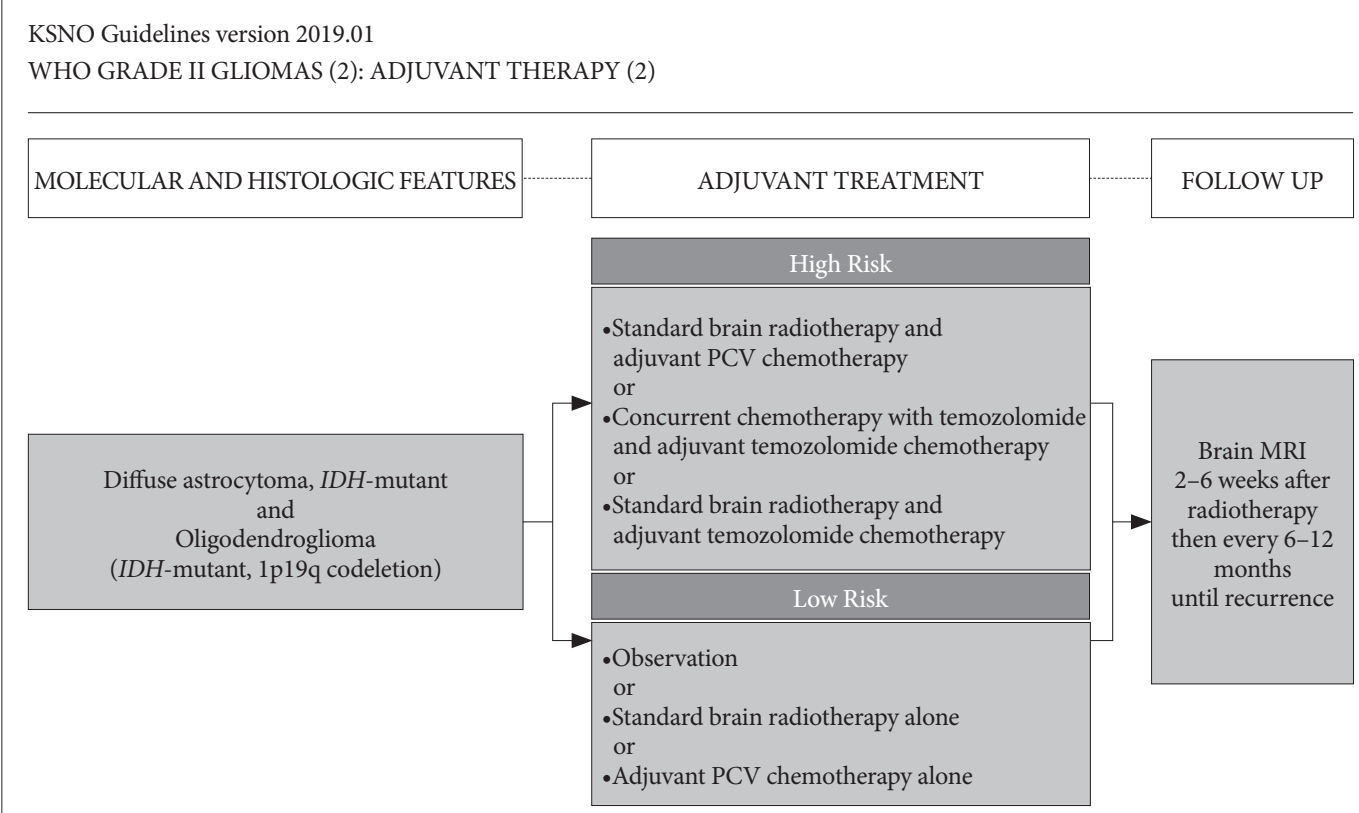

Fig. 3. Guideline for adjuvant therapy of patients with diffuse astrocytoma, IDH-mutant and oligodendroglioma. 1) The standard brain radiotherapy and adjuvant PCV (procarbazine+lomustine+vincristine) combination chemotherapy should be considered primarily for the high-risk group, 2) while observation with regular follow up should be considered for the low-risk group. IDH, isocitrate dehydrogenase; KSNO, Korean Society for Neuro-Oncology; PCV, procarbazine+lomustine+vincristine; MRI, magnetic resonance image.

II gliomas than those of WHO grade III and IV gliomas because WHO grade II gliomas have less contrast enhancement. Especially, recurrence or progression can be indistinguishable from pseudoprogression on MRI within the first 3 months after completion of radiotherapy. However, the following radiologic findings can suggest recurrence or progression as recommended by radiologic assessment of neurooncology (RANO) criteria for low grade gliomas [16]: 1) 25\% or more increase in the size of the lesion in fluid-attenuated inversion recovery (FLAIR) image and T2 weighted image, 2) any new lesion, and 3) clinical deterioration (not attributable to other non-tumor causes or steroid decrease) occurs simultaneously that strongly suggests true progression.

If recurrence or progression is suspected based on the above criteria, it is better to consider the following options: 1) undergoing biopsy, 2) performing functional radiologic study such as MR spectroscopy, MR perfusion, brain positron emission tomography (PET)-CT, or 3) checking MRI again and comparing changes that might be due to progression versus other causes. When recurrence of the tumor is suggested clinically and radiologically, surgical resection is always recommended, if feasible (Fig. 4). Even if recurrence of WHO grade II glioma has difficulty to achieve complete resection due to diffuse, multi-focal, or deep located lesion, surgical treatment can be considered to reduce the mass effect and improve neurological symptoms. The following systemic therapies can be considered for recurrent WHO grade II gliomas based on the physician's decision: cytotoxic chemotherapy including repeated PCV, target therapy, and/or immunotherapy, and so on. Also, radiotherapy can be considered for patients naïve to radiotherapy. However, for patients who have undergone prior radiotherapy, reirradiation can be considered, especially if the interval from prior radiotherapy is greater than 1 year, or if the new lesion is located outside the previously irradiated brain, or if the recurrence is small and geometrically favorable. Additionally, enrollment of clinical trials can be considered. Supportive treatment only can be considered for patients with poor performance status (Fig. 4).

\section{PRINCIPLES OF THE MANAGEMENT OF WHO GRADE II GLIOMAS}

\section{Brain imaging}

Many imaging modalities are available in neurooncology primarily to make treatment decisions in Korea. Imaging is always recommended to investigate emergent signs or symptoms. MRI of the brain (with and without contrast) is the gold standard modality to investigate brain tumors. It provides a static picture of brain tumors. It has a benefit in that it provides a reasonably good delineation of tumors. In MRI, high grade tumors and brain leptomeningeal metastases usually show enhancement while low-grade tumors usually do not. However, it has a limitation in that it is sensitive to movement. In addition, metallic objects can cause artifacts. Thus, patients 
KSNO Guidelines version 2019.01

WHO GRADE II GLIOMAS (3): RECURRENT DISEASE

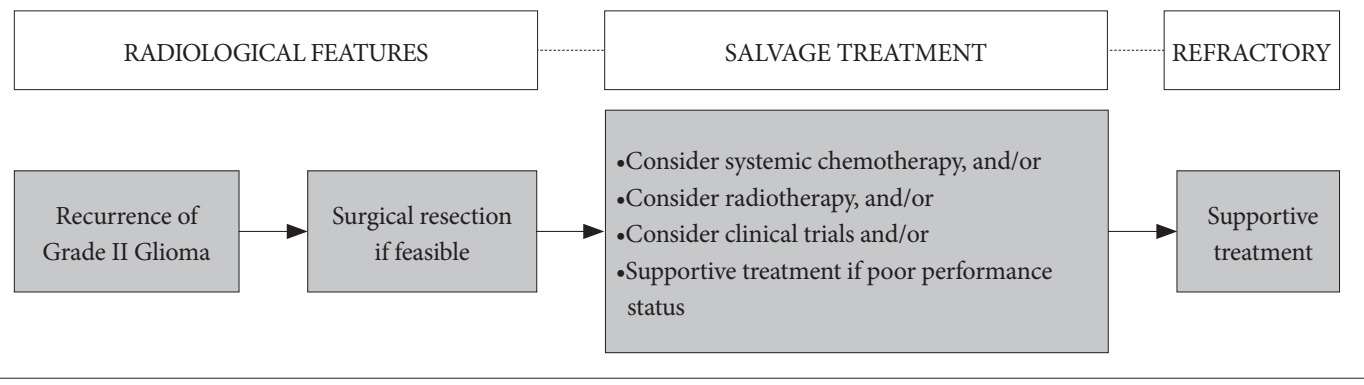

Fig. 4. Guideline for recurrent WHO grade II gliomas. Surgical resection is always recommended, even in the recurrence of grade II gliomas with difficulty in complete resection such as diffuse, multi-focal, or deep located lesion in order to reduce the mass effect and improve the neurological symptoms. After surgical resection, the following therapeutic options are considered: 1) systemic chemotherapy, and/or 2) reirradiation, and/or 3) enrollment of clinical trials and/or 4) supportive treatment if poor performance status. KSNO, Korean Society for Neuro-Oncology.

with implantable devices cannot receive an MRI. Moreover, claustrophobia and renal insufficiency may be problematic. Postoperative brain MRI should be performed within 24-72 hours after surgery for gliomas and other tumors to determine the extent of resection.

CT scan of the brain (with and without contrast) is usually considered for patients who cannot undergo an MRI. CT has a benefit of avoiding claustrophobia or implanted devices in the body. It is faster than an MRI. However, CT has a limitation in that it lacks resolution, especially for tumor located within the posterior fossa and for patients with renal insufficiency.

MR spectroscopy can be used to assess metabolites within tumor and normal tissues. It may be useful for differentiating tumors from radiation necrosis. It may also be helpful in grading tumors or assessing therapeutic response. The area showing the most abnormal features would be the best place for a biopsy. However, it has limitation for tumors near vessels, air space, or bone.

MR perfusion can be used to measure cerebral blood volume in tumors. It may be useful for differentiating the grade of tumor or tumor versus radiation necrosis. The area with the highest perfusion would be the best place for a biopsy. However, it has also limitation for tumors near vessels, air space, or bone, and small-volume lesions.

PET-CT using fluorodeoxyglucose (FDG) has a limitation for functional diagnosis of WHO grade II cerebral glioma due to high uptake of FDG in the brain with normal biologic metabolism. However, PET-CT using methionine, an essential amino acid, may be helpful for differentiating the grade of tumor or tumor versus radiation necrosis. Unfortunately, this technology is not commonly used in the clinical field in Korea. Further development of its application is necessary for casual use in clinical practice.

\section{Multidisciplinary care}

During treatment, most patients with WHO grade II cerebral glioma will be managed by various subspecialists. Close and regular communication among all providers across multi-disciplines is essential. Utilization of a brain tumor board or multidisciplinary clinic care models can facilitate interactions among various subspecialists. Ideally, allied health services (e.g., physical, occupational and speech therapies, nursing, psychology, and social services) should be included to optimize treatment plan recommendations.

As treatment proceeds, it is important for patient and his/ her family to understand the role of each team member. One attending physician who mainly cares for the patient should be determined as early as possible and the attending physician should contact the patient regularly for follow up. Additionally, the attending physician should facilitate referral to appropriate specialist.

The patient is strongly encouraged to participate in various clinical trials. Practitioners should discuss local, regional, and national options for which patients may be eligible, along with advantages and disadvantages of their participation. The center that treats neuro-oncology patients should encourage patients to participate in large collaborative trials in order to have another option for patients.

Throughout treatment, patient's health-related quality of life should remain the highest priority. It should guide clinical decision-making. While therapeutic response in radiologic study is a good indicator of successful therapy, other non-radiologic indicators of therapeutic response such as overall well-being, cognitive function, physical and motor functioning in day-to-day activities, communication ability, social functioning and family interactions, nutrition, pain control, long-term consequences of treatment, and psychological issues should also be considered. 
Patients should be informed of the possibility of pseudoprogression. Its approximate incidence and potential investigations may be needed if pseudoprogression is suspected. Close follow-up imaging, MR spectroscopy, PET-CT imaging, and repeat neurosurgical intervention including biopsy and surgical resection may be necessary if clinically indicated.

\section{Brain tumor surgery}

General principles of surgical resection of WHO grade II cerebral glioma are as follows: gross total resection when appropriate, minimal surgical morbidity, and accurate diagnosis. The following factors should be considered when deciding surgical resection: age, performance status, feasibility of decreasing the effect of mass with surgery, resectability, including number of lesions, location of lesions, time since last surgery in recurrent patients, new versus recurrent tumor, and timing of surgery in patient without symptoms [17]. Suspected pathology should also be considered with the following points: benign versus malignant, possibility of other non-cancer diagnoses, and projected natural history. For patients with IDH1 mutants, there is evidence suggesting that a supra-marginal resection is the most appropriate. It should include enhancing areas and T2/FLAIR areas when appropriate in terms of safe surgical approach, with the use of any or all surgical adjuncts possible [17]

Options of surgical resection include gross total resection where feasible, stereotactic biopsy, and open biopsy/debulking followed by planned observation or adjuvant therapy. To obtain the maximal safe resection, neuronavigation systems, intraoperative MRI or CT, intraoperative ultrasonography, fluorescence-guided surgery with 5-aminolevulinic acid, and intraoperative mapping techniques may be helpful. For histopathological diagnosis and genetic information, sufficient tissue should be sent to the pathologist for evaluating neuropathological and molecular correlates. When possible, frozen section analysis can assist intraoperative decision making. The tissue should be reviewed by an experienced neuropathologist.

Postoperative brain MRI should be performed within 24-72 hours after surgery for WHO grade II cerebral glioma to determine the extent of resection. The extent of resection should be judged based on postoperative imaging study. It should be used as a baseline to assess further therapeutic efficacy or tumor progression.

\section{Pathology examination}

Incorporation of relevant diagnostic markers, including histopathologic and molecular information described in the 2016 WHO Classification of Tumors of the CNS, should be considered as standard practice for tumor classification. Mo- lecular/genetic characterization complements standard histologic analysis, providing additional diagnostic and prognostic information that can greatly improve diagnostic accuracy, influence treatment selection, and possibly improve management decision-making.

For standard histopathologic examination of $\mathrm{WHO}$ grade II cerebral gliomas, basic histologic examination is performed based on description presented in the WHO Classification of Tumors of the CNS [4]. Interobserver discrepancies in histologic diagnosis and grading are recognized issues due to the inherently subjective nature of certain aspects of histopathologic interpretation (e.g., astrocytic vs. oligodendroglial morphology). In addition, surgical sampling does not always capture all relevant diagnostic features of morphologically heterogeneous tumors.

Through genetic and molecular testing, WHO grade II gliomas can be differentiated more accurately in terms of prognosis and response to different therapies in some instances. However, molecular/genetic characterization does not replace standard histologic assessment. It serves as a complementary approach to provide additional diagnostic and prognostic information that can enhance treatment selection. Although there are no identified targeted agents with demonstrate efficacy in WHO grade II cerebral glioma, the panel encourages molecular testing of tumors such as performing next generation sequencing because if a driver mutation is detected, it may be reasonable to treat the patient with a targeted therapy on a compassionate use basis. In addition, the patient may have more treatment options in the context of a clinical trial.

Several specific molecular tests play a valuable role in improving diagnostic accuracy and prognostic stratification that may inform treatment selection. IDH1 and IDH2 mutation testing is an essential part for workup of WHO grade II glioma. If the tumor has IDH-wildtype, the following molecular tests are strongly recommend: 1) EGFR amplification, 2) combined whole chromosome 7 gain and whole chromosome 10 loss, and 3) TERT promoter mutation. Codeletion of $1 \mathrm{p} 19 \mathrm{q}$ testing is also an essential part of molecular diagnostics for oligodendrogliomas. Therefore, $1 \mathrm{p} 19 \mathrm{q}$ codeletion testing should be considered to differentiate astrocytoma from oligodendrogliomas. ATRX mutation test and MGMT gene promoter methylation test are also helpful for workup of WHO grade II gliomas.

\section{Radiotherapy}

The optimal timing for radiotherapy after surgical resection has not been established yet in WHO grade II gliomas. No study has shown that delay in radiotherapy can decrease survival. However, if adjuvant radiotherapy is indicated for 
patients, we recommend radiotherapy to be initiated at postoperative 2-6 weeks after full patient recovery from surgical interventions.

Whenever radiotherapy is planned for patients with WHO grade II gliomas, pre- and post-operative MRIs should be used to define all target volumes including gross and clinical tumor volume (GTV and CTV). Additional MRI at the time of radiotherapy simulation can be used to account for changes in surgical cavity or lesions. Planning CT-based 3-dimensional calculation of dose distribution should be used at any circumstance. The GTV should encompass preoperative tumor bed and high T2/FLAIR signals on postoperative MRI. Preoperative tumor bed should not be directly delineated on the registered preoperative MRI on the planning CT for patients undergoing surgical resection. An expansion of 1-2 cm CTV delineation to account for subclinical tumor infiltration should be utilized. Simple expansion from the GTV should be avoided. CTV should always be modified based on anatomical barriers for tumor infiltration. A margin of 3-5 mm from the CTV is usually recommended to create planning target volume (PTV) to account for errors from image-registration and daily set-up of patients. However, the margin can also be reduced if daily image-guidance is performed.

A total dose of 45-54 Gy is recommended as standard radiotherapy using a daily fraction of 1.8-2.0 Gy. Field reduction is not usually performed. Dose-escalation up to $60 \mathrm{~Gy}$ can be considered for $I D H$-wildtype WHO grade II gliomas considering their poor prognosis and aggressive nature. When intensity-modulated radiotherapy (IMRT) is used to avoid critical organs, simultaneous integrated boost (SIB) techniques can be used. Most patients with grade II glioma are usually considered for standard radiotherapy. However, in rare settings of fragile patients with old age or poor performance, hypofractionated radiotherapy in 1-4 weeks can be considered. The following regimens can be used: $40.05 \mathrm{~Gy} / 15$ fractions, $34 \mathrm{~Gy} / 10$ fractions, $50 \mathrm{~Gy} / 20$ fractions, and 25 Gy/5 fractions. Of note, these regimens are adopted from the experience in high-grade gliomas and evidence supporting their use in low-grade gliomas is lacking. Absolute cumulative dose limits and optimal interval between radiotherapy sessions for WHO grade II glioma remain unestablished. Delivery, dose, fraction, target volume, and techniques for reirradiation should be decided by brain tumor expert and radiation oncologist through multidisciplinary discussion whenever feasible.

\section{DISCUSSION}

This is the third practical guideline for CNS tumors which was developed by KSNO Guideline Working Group. Treat- ment of newly diagnosed WHO grade II gliomas is more controversial than other gliomas in neurooncological fields due to heterogeneity of these tumors, their variability in natural history, concern regarding morbidity of treatment, and absence of proven overall survival benefit from any known treatment [18]. There is also still unresolved issue that interobserver variation in distinction between WHO grades II and III is notoriously large, adding to the notion of a lack of relevance of WHO grading scale for individual glioma patients [18].

Historically, PCV chemotherapy is one of widely studied treatments for WHO grade II gliomas. Favorable responses seen with a combination of PCV in anaplastic oligodendrogliomas can lead to the evaluation of this combination in low grade oligodendrogliomas and oligoastrocytomas $[19,20]$ and eventually all histologies of diffuse low grade gliomas, as included in the Radiation Therapy Oncology Group (RTOG) 9802. With this background, RTOG 9802 was designed with two cohorts: 1) a high-risk group randomized to adjuvant chemotherapy or no adjuvant therapy after conventional radiation therapy; and 2) a low-risk group that was observed without radiation or chemotherapy [21]. The high-risk group in this study was defined as patients with diffuse gliomas (regardless of histology) who were 40 years or older with any extent of resection and patients who were 18 years or older whose tumors were less than completely resected [21]. In initial results of RTOG 9802, with a median follow-up of 5.9 years, those treated with PCV chemotherapy were found to have significantly prolonged PFS compared to patients who were treated with radiation alone. However, OS was not significantly prolonged in prespecified initial analysis [21]. Finally, the mature results from long-term follow-up also demonstrated that the difference in OS between these two arms was statistically significant. Patients treated with radiation therapy and PCV had a median OS of 13.3 years compared to 7.8 years for radiation alone group [18]. Although lomustine is not commonly used for solid cancer, it can be obtained by Korea Orphan and Essential drug Center. In addition, the Korean National Health Insurance System covers the use of PCV regimen for patients with WHO grade II gliomas.

The use of temozolomide is permitted for patients with WHO grade IV glioma and recurrent WHO grade III glioma in Korea, although it is still limited for those with WHO grade II gliomas. Over the past decade, PCV has been gradually replaced by temozolomide because temozolomide has an easier schedule and better tolerance by the patient. The RTOG 0424 trial, a phase II study of temozolomide-based chemoradiotherapy for high-risk low grade gliomas, showed that the 3-year OS rate of $73.1 \%$ for their cohort was higher than that reported for historical controls and the study-hy- 
pothesized rate of $65 \%$ [22]. In the trial, patients with highrisk were treated with radiation therapy (54 Gy in 30 fractions) and concurrent and adjuvant temozolomide. Up to date, several clinical trials have reported the effect of temozolomide on low grade gliomas $[10,14,22,23]$. Therefore, this guideline recommends temozolomide chemotherapy for the newly diagnosed WHO grade II diffuse astrocytoma and high-risk oligodendrogliomas, even if this treatment is limited in Korea.

In this guideline, "high-risk" low grade gliomas patients are simply defined as those of younger than 40 years old and having undergone gross total resection of tumors. However, the "high-risk versus low-risk" classification has no means of representing the exact science. In the "low-risk" cohort of RTOG 9802, three prognostic factors predicted decreased PFS: 1) preoperative tumor diameter $\geq 4 \mathrm{~cm} ; 2$ ) astrocytoma or oligoastrocytoma histological subtype; and 3) residual tumor $\geq 1 \mathrm{~cm}$ on MRI [20]. In the EORTC 22033 trial, at least one of the following criteria need to be present for classification of "high-risk" low grade gliomas: 1) age $\geq 40$ years, 2) radiologically proven progressive lesion, 3 ) new or worsening neurological symptoms other than seizures only (focal deficits, signs of raised intracranial pressure, mental deficits), or the presence of intractable seizures [14]. In the RTOG 0424 trial, patients having 3 or more of the following risk factors for recurrence were classified as "high-preoperative risk": 1) age $\geq 40$ years, 2) astrocytoma histology, 3) bi-hemispherical tumor, 4) tumor diameter of $\geq 6 \mathrm{~cm}$, or a preoperative neurological dysfunction [22]. A recent meta-analysis of four large randomized trials from the pre-molecular era (including RTOG 9802) showed four factors related to worse OS: 1) the presence of baseline neurological deficits, 2) a shorter time since first symptoms (<30 weeks), 3 ) an astrocytic tumor type, and 4) tumors larger than $5 \mathrm{~cm}$ in diameter [24]. Therefore, from a more practical perspective, the decision of treating with adjuvant chemotherapy should be based on both clinical features and molecular findings during the course of the disease.

In pathological diagnosis, there is still confusion regarding whether all diffuse gliomas (diffuse astrocytomas and oligodendrogliomas) need to be analyzed for $1 \mathrm{p} 19 \mathrm{q}$ status to meet WHO diagnostic requirements. The 2016 Blue Book on CNS tumors [4] states that the presence of an astrocytic "component" is compatible with the diagnosis of oligodendroglioma if it shows $1 \mathrm{p} 19 \mathrm{q}$ codeletion and IDH mutation. This implies that only oligodendrogliomas and mixed oligodendroglioma-astrocytoma tumors need to be analyzed for $1 \mathrm{p} 19 \mathrm{q}$ codeletion, whereas histologically pure astrocytomas do not. On the other hand, according to the 2016 CNS WHO review article published in Acta Neuropathologica [3], a diffuse glioma that is histologically astrocytic but has $1 \mathrm{p} 19 \mathrm{q}$ codeletion and IDH mutation necessitates a diagnosis of oligodendroglioma. This implies that $1 \mathrm{p} 19 \mathrm{q}$ analysis is required for all cases of $I D H$-mutated diffuse glioma, including pure astrocytic tumors. Neuropathologists who are experts in practice have clarified that diffuse gliomas with $I D H$ mutation and astrocytoma morphology do not need reflex testing for $1 \mathrm{p} 19 \mathrm{q}$ loss. They can be designated as astrocytoma, $I D H$-mutant if ATRX and p53 immunohistochemistry findings support the diagnosis [25].

In terms of radiological assessment for low grade gliomas, there are something difficulties to apply it in clinical fields because low grade gliomas are usually measured in T2/ FLAIR rather than contrast enhancement as these tumors rarely enhance. In addition, as responses to treatment are often relatively modest, minor response criteria that are characterized by a decrease in T2/FLAIR tumor of $25 \%$ to $50 \%$ was introduced. Although T2/FLAIR provides the clearest and the most reproducible definition of low-grade gliomas, distinguishing tumor from radiation-induced changes, postsurgical changes, demyelination, ischemic injury, and other comorbid events can be difficult. As validated imaging modalities that can more accurately reflect tumor burden are developed, these criteria can be revised. For low grade gliomas, clinical outcome assessments such as neurocognitive function, quality of life, and seizure control play important roles in determining the response to treatment. The RANO group has recently proposed guidelines for using seizure control as a metric to assess the efficacy of tumor treatment in clinical trials for low grade gliomas by using a composite score of seizure classification, frequency, outcome, and severity [26]. Another challenge in determining response and progression of WHO grade II gliomas is the difficulty in accurately measuring the tumor using only two-dimension. There is ongoing work to determine if measuring T2/FLAIR volume is more accurate in determining changes in tumor burden and whether newer approaches such as determining changes in tumor volume growth trajectory can be more reliable and sensitive measure of response [27].

Limitations of this KSNO guideline for WHO grade II cerebral gliomas are not different from those of the KSNO guideline for other brain tumors such as WHO grade III and IV. The major weakness of this guideline is limited application to Korean patients with WHO grade II gliomas due to unique medical atmosphere of Korea. Therefore, it is less helpful for physicians treating patients outside of Korea. To use this guideline globally, Asian countries including Japan and China should have a comprehensive network for brain tumor management. They should cooperate and share their guidelines. Further, it will be helpful for Asian countries to establish a 
global guideline that is commonly applicable to these countries. The next hurdle to be overcome is molecular and genetic test for WHO grade II gliomas and whole brain tumors. Genetic information is now rapidly changing, making it difficult to establish definite guideline for clinical practice. The cIMPACT-NOW is a notable example that shows rapid change in molecular diagnosis of brain tumors $[9,28,29]$. Third, this guideline does not include medical management. In clinical practice, physicians are still struggling to manage the mass effect, brain edema, radiation necrosis, seizures, endocrine dysfunctions, fatigues, psychological disorders such as depression and anxiety, and venous thromboembolism [17]. However, there is no consensus for steroid therapy, the use of antiepileptic drugs, or antipsychotic drugs for these patients. Finally, this guideline did not include all WHO grade II cerebral gliomas. Only patients with diffuse astrocytoma and oligodendroglioma were subjects for this guideline. Other WHO grade II cerebral gliomas such as ependymoma, pilomyxoid astrocytoma, and pleomorphic xanthoastrocytoma were not included in this guideline, neither gliomas of the spinal cord. Therefore, the KSNO's Guideline Working Group has plans to continue the process of updating guidelines so that limitations of this version can be improved.

\section{CONCLUSIONS}

There was no practical guideline for the management of brain tumor patients. Thus, the KSNO developed this guideline that could be used by physicians under unique medical circumstances in Korea. The KSNO Guideline Working Group composed of 35 multidisciplinary medical experts in Korea prepared "The KSNO guideline for WHO grade II cerebral glioma in adults: Version 2019.01" as the third guideline following the KSNO Guideline for WHO grade IV and III glioma.

In summary, WHO grade II gliomas in the brain should be treated by maximal safe resection if feasible, followed by radiotherapy and/or chemotherapy according to individual molecular and histopathological features of tumors as well as clinical status of patients. As data emerging in the past few years have led to significant changes in the diagnosis, categorization, and treatment of WHO grade II gliomas, we plan to update this guideline consistently. Consecutive guideline for other brain tumors such as WHO grade I gliomas, brain metastasis, and meningiomas will also be published by the KSNO Guideline Working Group.

\section{Conflicts of Interest}

The authors have no potential conflicts of interest.

\section{Acknowledgments}

This protocol can be applied for adult patients with WHO grade II glio- mas of the brain. We especially appreciate the following individuals for their help: Chan Woo Wee, M.D. (Department of Radiation Oncology, Seoul National University Bundang Hospital) for describing the principle of radiotherapy as well as systemic review of radiotherapy for WHO grade II gliomas, Soon-Tae Lee, M.D. (Department of Neurology, Seoul National University Hospital) for describing the principle of multidisciplinary care, Tae Hoon Rho, M.D. (Department of Neurosurgery, AJou University Hospital) for performing systemic review of chemotherapy for WHO grade II gliomas, Je Beom Hong, M.D. (Department of Neurosurgery, Kangbuk Samsung Hospital) for performing systemic review of surgical treatment for WHO grade II gliomas, and Hyuk-Jin Oh, M.D. (Department of Neurosurgery, Soonchunhyang University Cheonan Hospital) for performing systemic review of treatment for recurrent WHO grade II gliomas.

\section{Author Affiliations}

${ }^{1}$ Division of Neurooncology and Department of Neurosurgery, Samsung Changwon Hospital, Sungkyunkwan University School of Medicine, Changwon, Korea; ${ }^{2}$ Department of Neurosurgery, Seoul National University Bundang Hospital, Seoul National University College of Medicine, Seongnam, Korea; ${ }^{3}$ Department of Radiation Oncology, Seoul National University Bundang Hospital, Seoul National University College of Medicine, Seongnam, Korea; ${ }^{4}$ Department of Neurosurgery, Ajou University Hospital, Ajou University School of Medicine, Suwon, Korea; ${ }^{5}$ Department of Neurosurgery, Kangbuk Samsung Hospital, Sungkyunkwan University School of Medicine, Seoul, Korea; ${ }^{6}$ Department of Neurosurgery, Soonchunhyang University Cheonan Hospital, Soonchunhyang University College of Medicine, Cheonan, Korea; ${ }^{7}$ Department of Neurosurgery, Severance Hospital, Yonsei University College of Medicine, Seoul, Korea; ${ }^{8}$ Department of Neurosurgery, Korea University Anam Hospital, Korea University College of Medicine, Seoul, Korea; ${ }^{9}$ Department of Neurosurgery, Samsung Medical Center, Sungkyunkwan University School of Medicine, Seoul, Korea; ${ }^{10}$ Department of Radiation Oncology, St. Vincent's Hospital, College of Medicine, The Catholic University of Korea, Seoul, Korea; ${ }^{11}$ Department of Pathology, Severance Hospital, Yonsei University College of Medicine, Seoul, Korea; ${ }^{12}$ Division of Medical Oncology, Department of Internal Medicine, Seoul National University Bundang Hospital, Seoul National University College of Medicine, Seongnam, Korea; ${ }^{13}$ Department of Radiology and Research Institute of Radiology, University of Ulsan College of Medicine, Asan Medical Center, Seoul, Korea; ${ }^{14}$ Department of Neurosurgery, Seoul St. Mary's Hospital, College of Medicine, The Catholic University of Korea, Seoul, Korea; ${ }^{15}$ Clinic of Pediatric Oncology, National Cancer Center, Goyang, Korea; ${ }^{16}$ Department of Neurosurgery, Konkuk University Medical Center, Konkuk University School of Medicine, Seoul, Korea.; ${ }^{17}$ Department of Neurosurgery, Dong-A University Hospital, DongA University College of Medicine, Busan, Korea; ${ }^{18}$ Department of Neurosurgery, St. Vincent's Hospital, College of Medicine, The Catholic University of Korea, Seoul, Korea; ${ }^{19}$ Department of Neurosurgery, Incheon St. Mary's Hospital, College of Medicine, The Catholic University of Korea, Incheon, Korea; ${ }^{20}$ Department of Radiation Oncology, Yonsei Cancer Center, Yonsei University College of Medicine, Seoul, Korea; ${ }^{21}$ Department of Radiation Oncology, Ewha Women's University Mokdong Hospital, Ewha Women's University School of Medicine, Seoul, Korea; ${ }^{22}$ Department of Neurology, Seoul National University Hospital, Seoul National University College of Medicine, Seoul, Korea; ${ }^{23}$ Department of Radiation Oncology, Seoul St. Mary's Hospital, College of Medicine, The Catholic University of Korea, Seoul, Korea; ${ }^{24}$ Department of Pathology, Seoul St. Marry's Hospital, College of Medicine, The Catholic University of Korea, Seoul, Korea; ${ }^{25}$ Department of Neurosurgery, Bundang CHA Medical Center, CHA University, Seongnam, Korea; ${ }^{26}$ Department of Neurosurgery, Chonnam National University Hwasun Hospital, Chonnam National University Medical School, Gwangju, Korea; ${ }^{27}$ Department of Pediatrics, Kangbuk Samsung Hospital, Sungkyunkwan University School of Medicine, Seoul, Korea; ${ }^{28}$ Department of Radiology, Seoul National University Hospital, Seoul National University College of Medicine, Seoul, Korea; ${ }^{29}$ Department of Pediatrics, Seoul National University Bundang Hospital, Seoul National University College of 
Medicine, Seongnam, Korea; ${ }^{30}$ Department of Radiation Oncology, Samsung Medical Center, Sungkyunkwan University School of Medicine, Seoul, Korea

\section{REFERENCES}

1. Ostrom QT, Gittleman H, Truitt G, Boscia A, Kruchko C, BarnholtzSloan JS. CBTRUS statistical report: primary brain and other central nervous system tumors diagnosed in the United States in 2011-2015. Neuro Oncol 2018;20(suppl_4):iv1-86.

2. Dho YS, Jung KW, Ha J, et al. An updated nationwide epidemiology of primary brain tumors in Republic of Korea, 2013. Brain Tumor Res Treat 2017;5:16-23.

3. Louis DN, Perry A, Reifenberger G, et al. The 2016 World Health Organization classification of tumors of the central nervous system: a summary. Acta Neuropathol 2016;131:803-20.

4. Louis DN, Ohgaki H, Wiestler OD, Cavenee WK. WHO classification of tumours of the central nervous system, revised. 4th ed. Lyon: International Agency for Research on Cancer; 2016.

5. Van den Bent MJ. Interobserver variation of the histopathological diagnosis in clinical trials on glioma: a clinician's perspective. Acta Neuropathol 2010;120:297-304.

6. Malzkorn B, Reifenberger G. Practical implications of integrated glioma classification according to the World Health Organization classification of tumors of the central nervous system 2016. Curr Opin Oncol 2016;28:494-501.

7. Weller M, Wick W, Aldape K, et al. Glioma. Nat Rev Dis Primers 2015;1:15017.

8. Louis DN, Perry A, Burger P, et al. International Society of Neuropathology--Haarlem consensus guidelines for nervous system tumor classification and grading. Brain Pathol 2014;24:429-35.

9. Brat DJ, Aldape K, Colman H, et al. cIMPACT-NOW update 3: recommended diagnostic criteria for "Diffuse astrocytic glioma, IDHwildtype, with molecular features of glioblastoma, WHO grade IV". Acta Neuropathol 2018;136:805-10.

10. Koo T, Lim DH, Seol HJ, et al. Impact of adjuvant treatments on survival in Korean patients with WHO grade II gliomas: KNOG 15-02 and KROG 16-04 intergroup study. J Neurooncol 2018;140:445-55.

11. Shaw E, Arusell R, Scheithauer B, et al. Prospective randomized trial of low- versus high-dose radiation therapy in adults with supratentorial low-grade glioma: initial report of a North Central Cancer Treatment Group/Radiation Therapy Oncology Group/Eastern Cooperative Oncology Group study. J Clin Oncol 2002;20:2267-76.

12. Van den Bent MJ, Afra D, de Witte O, et al. Long-term efficacy of early versus delayed radiotherapy for low-grade astrocytoma and oligodendroglioma in adults: the EORTC 22845 randomised trial. Lancet 2005;366:985-90.

13. Buckner JC, Shaw EG, Pugh SL, et al. Radiation plus procarbazine, CCNU, and vincristine in low-grade glioma. N Engl J Med 2016;374:1344-55.

14. Baumert BG, Hegi ME, van den Bent MJ, et al. Temozolomide chemotherapy versus radiotherapy in high-risk low-grade glioma (EORTC
22033-26033): a randomised, open-label, phase 3 intergroup study. Lancet Oncol 2016;17:1521-32.

15. Kim YZ, Kim CY, Lim J, et al. The Korean Society for Neuro-Oncology (KSNO) Guideline for Glioblastomas: Version 2018.01. Brain Tumor Res Treat 2019;7:1-9.

16. Wen PY, Chang SM, Van den Bent MJ, Vogelbaum MA, Macdonald DR, Lee EQ. Response assessment in neuro-oncology clinical trials. J Clin Oncol 2017;35:2439-49.

17. National Comprehensive Cancer Network. NCCN clinical practice guidelines in oncology, central nervous system cancers. Version 1.2019. (Accessed August 1, 2019, at https://www.nccn.org/professionals/physician_gls/default.aspx\#cns.)

18. Van den Bent MJ. Practice changing mature results of RTOG study 9802: another positive PCV trial makes adjuvant chemotherapy part of standard of care in low-grade glioma. Neuro Oncol 2014;16:1570-4.

19. Buckner JC, Gesme D Jr, O’Fallon JR, et al. Phase II trial of procarbazine, lomustine, and vincristine as initial therapy for patients with lowgrade oligodendroglioma or oligoastrocytoma: efficacy and associations with chromosomal abnormalities. J Clin Oncol 2003;21:251-5.

20. Soffietti R, Rudà R, Bradac GB, Schiffer D. PCV chemotherapy for recurrent oligodendrogliomas and oligoastrocytomas. Neurosurgery 1998;43:1066-73.

21. Shaw EG, Wang M, Coons SW, et al. Randomized trial of radiation therapy plus procarbazine, lomustine, and vincristine chemotherapy for supratentorial adult low-grade glioma: initial results of RTOG 9802. J Clin Oncol 2012;30:3065-70.

22. Fisher BJ, Hu C, Macdonald DR, et al. Phase 2 study of temozolomidebased chemoradiation therapy for high-risk low-grade gliomas: preliminary results of Radiation Therapy Oncology Group 0424. Int J Radiat Oncol Biol Phys 2015;91:497-504.

23. Gwak HS, Yee GT, Park CK, et al. Temozolomide salvage chemotherapy for recurrent anaplastic oligodendroglioma and oligo-astrocytoma. J Korean Neurosurg Soc. 2013;54:489-95.

24. Gorlia T, Wu W, Wang M, et al. New validated prognostic models and prognostic calculators in patients with low-grade gliomas diagnosed by central pathology review: a pooled analysis of EORTC/RTOG/ NCCTG phase III clinical trials. Neuro Oncol 2013;15:1568-79.

25. Yeaney GA, Brat DJ. What every neuropathologist needs to know: update on cIMPACT-NOW. J Neuropathol Exp Neurol 2019;78:294-6.

26. Avila EK, Chamberlain M, Schiff D, et al. Seizure control as a new metric in assessing efficacy of tumor treatment in low-grade glioma trials. Neuro Oncol 2017;19:12-21.

27. Van den Bent MJ, Smits M, Kros JM, Chang SM. Diffuse infiltrating oligodendroglioma and astrocytoma. J Clin Oncol 2017;35:2394-401.

28. Louis DN, Aldape K, Brat DJ, et al. Announcing cIMPACT-NOW: the consortium to inform molecular and practical approaches to CNS Tumor taxonomy. Acta Neuropathol 2017;133:1-3.

29. Louis DN, Giannini C, Capper D, et al. cIMPACT-NOW update 2: diagnostic clarifications for diffuse midline glioma, H3 K27M-mutant and diffuse astrocytoma/anaplastic astrocytoma, IDH-mutant. Acta Neuropathol 2018;135:639-42. 